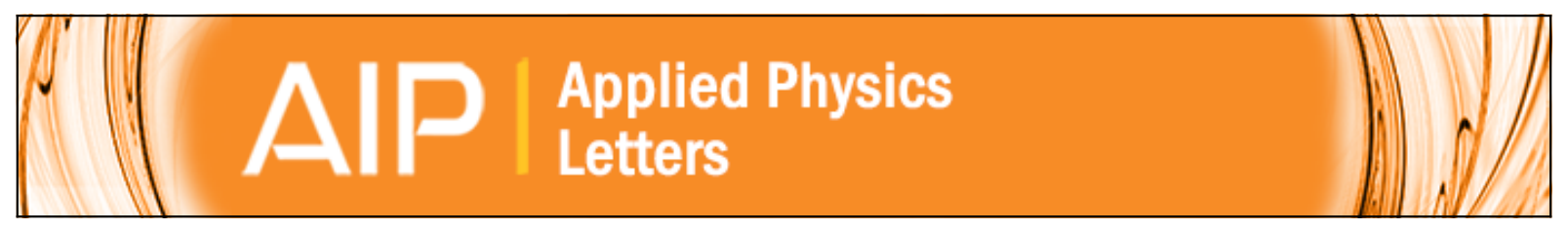

\title{
Short range scattering effect of InAs quantum dots in the transport properties of two dimensional electron gas
}

E. S. Kannan, Gil-Ho Kim, Sanjeev Kumar, I. Farrer, D. A. Ritchie, Jun Ho Son, Jeong Min Baik, Jong-Lam Lee, D. H. Youn, and Kwang-Yong Kang

Citation: Applied Physics Letters 90, 152110 (2007); doi: 10.1063/1.2720704

View online: http://dx.doi.org/10.1063/1.2720704

View Table of Contents: http://scitation.aip.org/content/aip/journal/apl/90/15?ver=pdfcov

Published by the AIP Publishing

\section{Articles you may be interested in}

Effects of scattering on two-dimensional electron gases in InGaAs/InAIAs quantum wells J. Appl. Phys. 112, 023713 (2012); 10.1063/1.4737777

The influence of charged InAs quantum dots on the conductance of a two-dimensional electron gas: Mobility vs. carrier concentration

Appl. Phys. Lett. 99, 223510 (2011); 10.1063/1.3665070

Short range scattering mechanism of type-II GaSb/GaAs quantum dots on the transport properties of twodimensional electron gas

J. Appl. Phys. 108, 043702 (2010); 10.1063/1.3467520

Effects of two-dimensional electron gas on the optical properties of InAs/GaAs quantum dots in modulationdoped heterostructures

Appl. Phys. Lett. 86, 021916 (2005); 10.1063/1.1849853

Transport properties of two-dimensional electron gases containing InAs self-assembled dots Appl. Phys. Lett. 73, 2468 (1998); 10.1063/1.122484

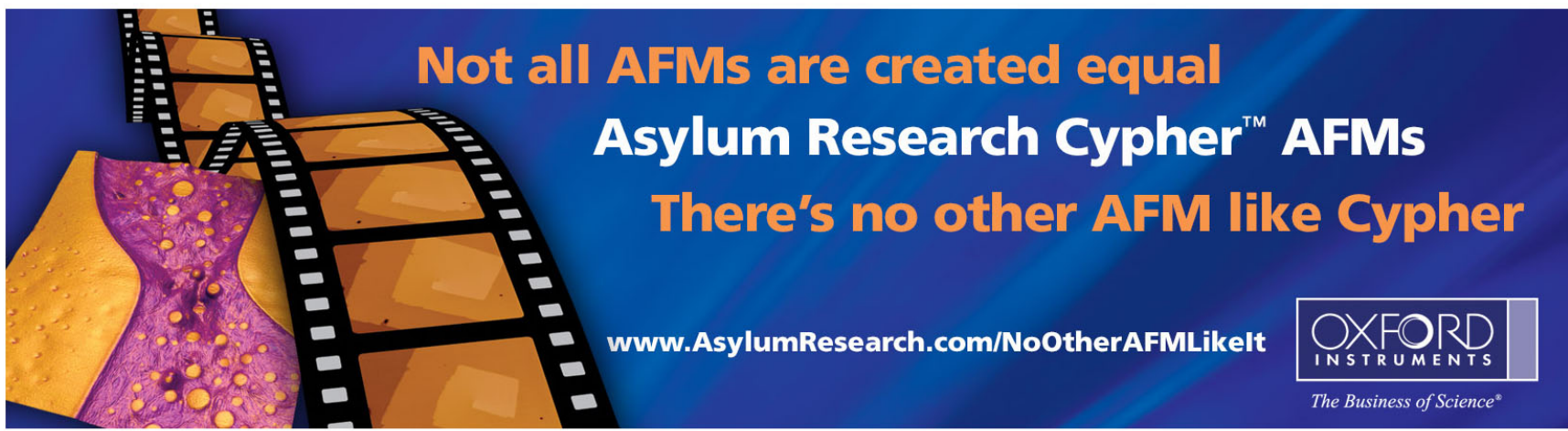




\title{
Short range scattering effect of InAs quantum dots in the transport properties of two dimensional electron gas
}

\author{
E. S. Kannan, Gil-Ho Kim, ${ }^{\text {a) }}$ and Sanjeev Kumar \\ School of Information and Communication Engineering, Sungkyunkwan University, Suwon 440-746, Korea \\ and SKKU Advanced Institute of Nanotechnology (SAINT), Sungkyunkwan University, Suwon 440- \\ 746, Korea \\ I. Farrer and D. A. Ritchie \\ Cavendish Laboratory, Madingley Road, Cambridge CB3 OHE, United Kingdom \\ Jun Ho Son, Jeong Min Baik, and Jong-Lam Lee \\ Department of Materials Science and Engineering, Pohang University of Science and Technology, Pohang \\ 790-784, Korea \\ D. H. Youn and Kwang-Yong Kang \\ IT Components \& Materials Technology Research Division, Electronics and Telecommunications Research \\ Institute (ETRI), Daejeon 305-350, Korea
}

(Received 21 December 2006; accepted 8 March 2007; published online 12 April 2007)

\begin{abstract}
Short range interaction between two dimensional electron gas (2DEG) and InAs quantum dots embedded in the GaAs/AlGaAs quantum well is investigated as a function of carrier density. At low carrier density the interaction is significantly characterized by a transport to quantum lifetime ratio of less than 5. However, with an increase in carrier density, quantum lifetime is observed to undergo a sharp transition from 0.17 to $0.25 \mathrm{ps}$. This is attributed to the screening of short range repulsive scattering due to InAs quantum dots by the 2DEG. (C) 2007 American Institute of Physics. [DOI: 10.1063/1.2720704]
\end{abstract}

Study of scattering in two dimensional electron gas (2DEG) is important for optimizing the performance of high electron mobility transistors. Extensive studies on the effect of scattering in the transport properties of $2 \mathrm{DEG}$ is carried out by measuring transport and quantum lifetime for modulation doped GaAs/AlGaAs heterostructure. ${ }^{1,2}$ Recently owing to the rapid development of quantum dot based devices such as quantum dot field effect transistor, quantum dot memory devices, and single photon detector, scattering effect of quantum dots on the $2 \mathrm{DEG}$ is studied with greater interest. $^{3-7}$ Some groups have attempted to study the effect of scattering due to InAs quantum dots in the transport properties of $2 \mathrm{DEG} .{ }^{8-10}$ In one of such studies, scattering of InAs quantum dots embedded in the vicinity of the GaAs channel is reported to affect the transport properties of the $2 \mathrm{DEG}$ such as mobility, transport, and quantum lifetimes when the thickness of the spacing layer between the 2DEG and the quantum dot is reduced. ${ }^{9}$ On the other hand, these transport properties of the 2DEG were enhanced as the InAs dot density was reduced when the thickness of the spacing layer and carrier density is maintained constant. ${ }^{10}$ However, there is scarcely any report on the scattering effect of InAs quantum dots of constant density embedded in AlGaAs/GaAs quantum well without any spacing layer.

In this letter, the short range interaction between $2 \mathrm{DEG}$ and InAs quantum dots embedded in the quantum well is investigated. The effect of short range scattering due to InAs quantum dots in the transport properties of $2 \mathrm{DEG}$ in the quantum well is studied for increasing carrier density. Carrier density is varied by changing the front gate voltage and the ability of 2DEG to screen the short range scattering is analyzed from the variation in transport and quantum lifetime. ${ }^{11}$

${ }^{a}$ Author to whom correspondence should be addressed; electronic mail: ghkim@skku.edu
The sample used for this study is a GaAs/AlGaAs single quantum well structure grown by molecular beam epitaxy. The heterostructure is grown on GaAs substrate and consists of an 18-nm-wide $\mathrm{GaAs} / \mathrm{Al}_{0.33} \mathrm{Ga}_{0.67} \mathrm{As}$ quantum well that is modulation doped on both sides using a $40 \mathrm{~nm}$ spacer layer. InAs quantum dots were then inserted in the quantum well at a distance of $9 \mathrm{~nm}$ from the lower half of the quantum well. Hall bars were fabricated using standard photolithographic technique. Shubnikov-de Hass oscillations ( $\mathrm{SdH}$ ) and Hall measurements were performed in a pumped liquid helium cryostat at a temperature of $1.4 \mathrm{~K}$ using a superconducting magnet capable of producing a magnetic field of $7 \mathrm{~T}$. During the entire measurement process, the channel current is maintained at $95 \mathrm{nA}$.

The study is carried out by changing the front gate voltage from -0.2 to $0.76 \mathrm{~V}$ to vary the carrier density in the quantum well. With increase in gate voltage, the frequency of $\mathrm{SdH}$ oscillation increased as carrier density increases. We observed a single frequency in the $\mathrm{SdH}$ oscillations indicating that only one subband is occupied (Fig. 1). Therefore the short range scattering effect of InAs quantum dots can be

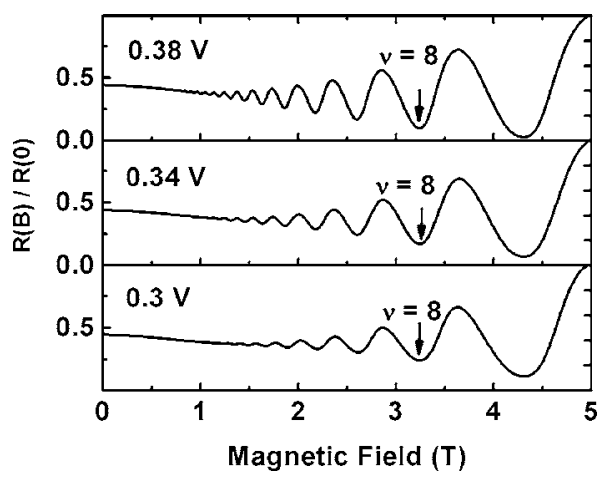

FIG. 1. Normalized SdH oscillations for 0.3, 0.34, and $0.38 \mathrm{~V}$ at $1.4 \mathrm{~K}$. 


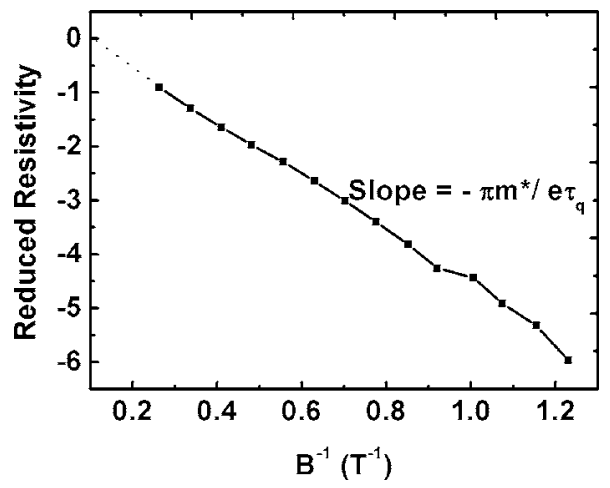

FIG. 2. Dingle plot corresponding to a gate voltage of $0.5 \mathrm{~V}$.

investigated by calculating the quantum lifetime using standard analytical technique. Carrier density measured from the low field $(0-0.4 \mathrm{~T})$ Hall slopes was found to increase from $3.07 \times 10^{11}$ to $6.34 \times 10^{11} \mathrm{~cm}^{-2}$ in the above mentioned gate voltage ranges and the mobility of the sample varied from $1.26 \times 10^{4}$ to $2.22 \times 10^{4} \mathrm{~cm}^{2} / \mathrm{V}$ s. These low mobility values are expected for a $2 \mathrm{DEG}$ containing InAs quantum dots. ${ }^{9}$ Moreover, longitudinal resistivity of $0.6-1 \mathrm{k} \Omega \mathrm{cm}^{-1}$ at $B=0$ for the range of carrier density studied indicates that repulsive scattering potential of inserted InAs dot structures affects the transport properties of the 2DEG significantly. ${ }^{9}$ However, the influence of the scattering phenomenon on the transport properties can be understood only from the analysis of transport and quantum lifetimes.

Transport lifetime $\left(\tau_{t}\right)$ is determined by using the classical equation $\tau_{t}=m^{*} / \rho n_{s} e^{2}$, where $m^{*}$ is the effective mass, $\rho$ is the zero field resistivity, and $n_{s}$ is the electron carrier density. Quantum lifetime $\left(\tau_{s}\right)$ is calculated by using the equation

$$
\Delta \rho=4 \rho_{0} X(T) \exp \left(\frac{-\pi}{\omega_{c} \tau_{q}}\right) \hbar,
$$

where $\rho_{0}$ is the zero field resistivity, $\omega_{c}$ is the cyclotron frequency, and the thermal damping factor $X(T)$ is given by

$$
X(T)=\frac{2 \pi^{2} k T / \hbar \omega_{c}}{\sinh \left(2 \pi^{2} k T / \hbar \omega_{c}\right)} .
$$

To obtain the Dingle plot, logarithm of $\Delta \rho / 4 \rho_{0} X(T)$ is plotted against $1 / B$ for various carrier densities. The resulting graph is linear and has an intercept at zero for infinite magnetic field which is a characteristic of a good Dingle plot, as shown in Fig. 2. ${ }^{2}$

Figure 3 shows the variation of transport and quantum lifetimes with carrier density. As carrier density is increased, the transport lifetime increased steadily from 0.3 to $0.8 \mathrm{ps}$.

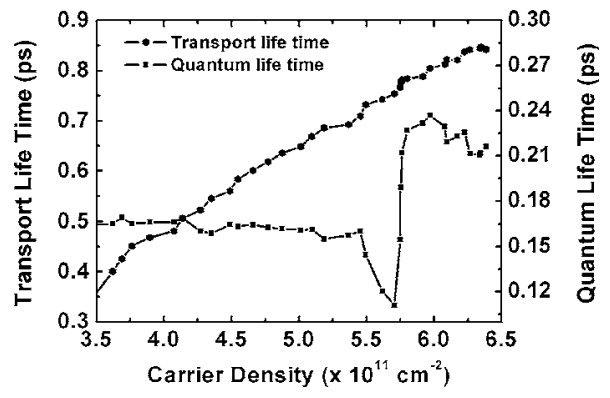

FIG. 3. Transport and quantum lifetime as a function of carrier density.

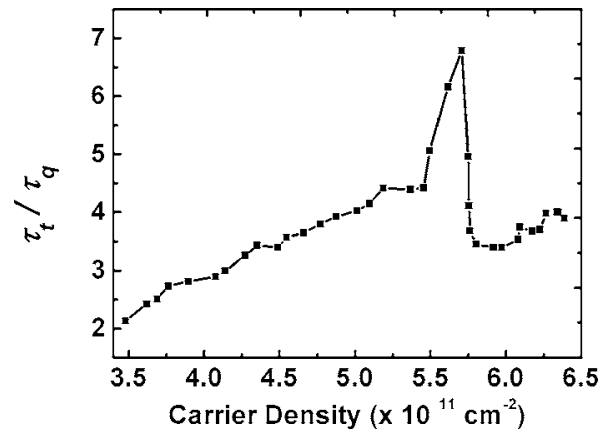

FIG. 4. Ratio of transport to quantum lifetime as a function of carrier density.

This behavior is typical for modulation doped heterostructure in which an increase in carrier density effectively screens the potential due to remote ionized donors. This screening of potential subsequently increases the transport lifetime and hence the mobility of the 2DEG also increases as carrier density is increased. ${ }^{12}$ Quantum lifetime, on the other hand, exhibits an interesting behavior. It remains almost constant at 0.17 ps until a carrier density of $5.5 \times 10^{11} \mathrm{~cm}^{-2}$ is reached and then decreases to $0.13 \mathrm{ps}$. Thereafter, there is a sharp increase in quantum lifetime attaining saturation at $0.25 \mathrm{ps}$ as carrier density is increased, as shown in Fig. 3. The ratio between $\tau_{t}$ and $\tau_{q}$ is found to vary within a range of 2-7, indicating that the dominant scattering mechanism is short range (Fig. 4). From the observed characteristics of transport and quantum lifetime, we hardly notice any correlation between them. In a recent investigation by Russ et al. on the nature of interaction between the 2DEG and InAs quantum dots separated by a tunnel barrier demonstrates that quantum dots act as uncorrelated scattering centers and enhance scattering due to resonant interaction with the $2 \mathrm{DEG} .{ }^{13}$ The potential from uncorrelated scattering centers cannot be treated by standard approximation methods to predict the variation in two dimensional density of states and hence the behavior of quantum lifetime. ${ }^{14}$ Therefore the absence of correlation between the two lifetimes observed in our study needs further detailed theoretical and experimental investigation.

On either side of the sharp transition region, quantum lifetime almost remains constant (Fig. 3). These observations suggest that the scattering rate due to short range potential can be controlled by varying the carrier density with the help of gate voltage to optimize our device performance. We present a plausible explanation for the abrupt change in the quantum lifetime with gate voltage observed in our sample. The potential candidate for the origin of short range scattering in our sample is the electrons in the quantum dots. Therefore by modeling the electrons as scattering centers the resultant screened potential can be approximated by the equation $^{15}$

$$
\langle U(q)\rangle^{2}=N_{\mathrm{qd}}\left(\frac{2 \pi e^{2}}{\kappa q}\right)^{2} F(q, d)^{2},
$$

where $N_{\text {qd }}$ is the density of electrons in the quantum dots, $q$ is the scattering wave number, $\kappa$ is the dielectric constant of GaAs, and $F(q, d)$ is the form factor which takes into account the finite extension of the 2DEG and the distance $d$ between the plane of the quantum dot and the $2 \mathrm{DEG} .{ }^{15} \mathrm{We}$ further assume that all parameters except $F(q, d)$ in Eq. (3) to remain constant. The validity of our assumption is supported 


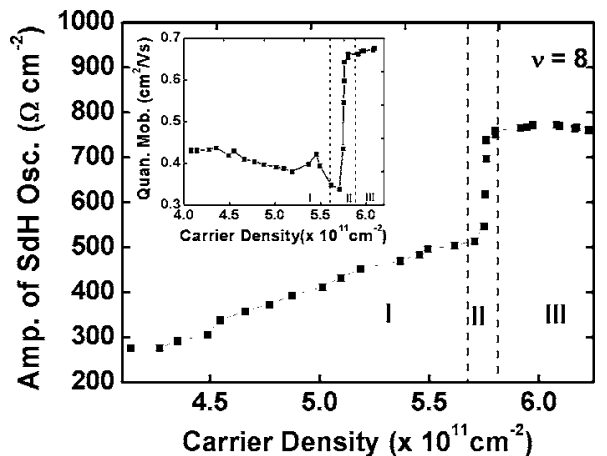

FIG. 5. Amplitude of SdH oscillation as a function of carrier density corresponding to filling factor $\nu=8$. The inset shows the variation of quantum mobility with carrier density.

by the fact that the sharp increase in quantum lifetime is observed for a narrow range of gate voltage during which number of electrons in the dots is not expected to vary much. Secondly, for two dimensional systems the screening is independent of $q$ for the entire range of scattering wave vectors $\left(0 \leqslant q \leqslant 2 k_{F}\right)$. As the front gate voltage is gradually increased, the wave function of the 2DEG localized around the quantum dots shifts toward the barrier interface. Neglecting finite extension of the $2 \mathrm{DEG}$, the form factor is given by ${ }^{16}$

$$
F(q, d)=e^{-2 q d} \text {. }
$$

The form factor undergoes an abrupt transition from 1 to 0.025 as the wave function shifts from the quantum dots $(d$ $=0)$ to the edge of the barrier $(d=9 \mathrm{~nm})$ when gate voltage is increased from 0.3 to $0.4 \mathrm{~V}$. This sharp reduction in $F(q, d)$ in turn decreases the scattering time in accordance with the equation (Stern-Howard model) ${ }^{15}$

$$
\frac{1}{\tau_{i}}=\frac{1}{2 \pi \hbar \varepsilon_{F}} \int_{0}^{2 k_{F}} d q \frac{q^{2}}{\sqrt{4 k_{F}^{2}-q^{2}}} \frac{\langle|U(q)|\rangle^{2}}{\varepsilon(q)^{2}} .
$$

This decrease in scattering time enhances the quantum lifetime $\left(\tau_{q}\right)$, which is related to the imaginary part of the single particle self-energy function $\left(\Gamma_{s}\right)$ by

$$
\Gamma_{s}=\frac{\hbar}{\tau_{q}},
$$

$\Gamma_{s}$ determines the broadening of the Landau levels due to the interaction between the 2DEG and the quantum dots. ${ }^{1}$ Therefore the increase in quantum lifetime reduces the broadening of Landau levels. This has the effect of increasing the resolution of $\mathrm{SdH}$ oscillation in the low magnetic field region. ${ }^{10}$ Careful investigation of Fig. 1 in our sample shows that as gate voltage is progressively increased from 0.32 to $0.38 \mathrm{~V}$, $\mathrm{SdH}$ oscillations begin to develop at fields less than $1 \mathrm{~T}$. Hence the sharp increase in quantum lifetime is attributed to the screening of short range scattering by the 2DEG due to its increased distance from the scattering centers. This explains the transition of quantum lifetime from 0.17 to $0.11 \mathrm{ps}$ as carrier density is varied from 5.5 $\times 10^{11}$ to $5.65 \times 10^{11} \mathrm{~cm}^{-2}(0.3-0.38 \mathrm{~V})$ (Fig. 3).

To gain further insight into this phenomenon, a plot of change in amplitude of SdH oscillation corresponding to filling factor $\nu=8$ is plotted against the carrier density in Fig. 5. This graph is characterized by three regions, namely, I, II, and III. In region I, there is a gradual increase in amplitude with increase in carrier density and in region II there is a sharp increase in amplitude for fractional change in carrier density followed by region III where the amplitude almost remains constant with moderate increase in carrier density.

The gradual increase in amplitude observed in region $\mathrm{I}$ is due to the increase in carrier density of the sample. As the carrier density is increased, eigenstates corresponding to higher Landau levels get filled up which tends to increase the fluctuation in the density of states. Therefore, the observed increase in amplitude in region I corresponding to filling factor $\nu=8$ in Fig. 5 is attributed to the increase in carrier density. The inset in Fig. 5 gives a clear picture of this scenario. We can observe that quantum mobility in region I decreased gradually in contrast to the amplitude of $\mathrm{SdH}$ oscillation. This indicates that the increase in amplitude is not due to the screening of short range scattering of InAs quantum dots.

The onset of screening of short range scattering by the 2DEG is characterized by sharp increase in amplitude for a fractional increase in carrier density in region II. The screening effect is also evident from Fig. 1, where the amplitude of $\mathrm{SdH}$ oscillations is found to increase even though the frequency of oscillations remains almost constant. As already explained, this is due to the gate voltage induced screening of short range scattering potential. In region III, the slight decrease in amplitude of $\mathrm{SdH}$ oscillation may be attributed to the increase in interface roughness scattering as 2DEG gets closer to the edge of the quantum well.

In conclusion, the scattering effect of InAs quantum dot embedded in GaAs quantum well is investigated. For a particular narrow range gate voltage and carrier density, the amplitude of $\mathrm{SdH}$ oscillations and quantum lifetime increased significantly. This is attributed to the screening of scattering potential due to InAs quantum dots by the $2 \mathrm{DEG}$. These observations suggest that the short range interaction between InAs quantum dots and the $2 \mathrm{DEG}$ can be controlled by varying the gate voltage.

This work was supported by the Ministry of Information and Communication, Republic of Korea, under Project No. A1100-0601-0110.

${ }^{1}$ B. Das, S. Subramniam, M. R. Melloch, and D. C. Miller, Phys. Rev. B 47, 15 (1993).

${ }^{2}$ S. Das Sarma and Frank Stern, Phys. Rev. B 32, 12 (1985).

${ }^{3}$ L. Hsu and W. Walukiewicz, Appl. Phys. Lett. 80, 2508 (2002).

${ }^{4}$ F. Stern, Phys. Rev. Lett. 18, 546 (1967).

${ }^{5}$ T. Wang, J. Bai, S. Sakai, Y. Ohno, and H. Ohno, Appl. Phys. Lett. 76, 2737 (2000).

${ }^{6}$ G. Yusa and H. Sakaki, Appl. Phys. Lett. 70, 345 (1997).

${ }^{7}$ Zhiliang Yuan, Beata E. Kardynal, R. Mark Stevenson, Andrew J. Shields, Charlene J. Lobo, Ken Cooper, Neil S. Beattie, David A. Ritchie, and Michael Pepper, Science 292, 102 (2002).

${ }^{8}$ C. I. Duruoz, R. M. Clarke, C. M. Marcus, and J. S. Harris, Jr., Nanotechnology 7, 372 (1996).

${ }^{9}$ H. Sakaki, G. Yusa, T. Someya, Y. Ohna, T. Noda, H. Akiyama, Y. Kadoya, and H. Noge, Appl. Phys. Lett. 67, 3444 (1995).

${ }^{10}$ G. H. Kim, D. A. Ritchie, M. Pepper, G. D. Lian, J. Yuan, and L. M. Brown, Appl. Phys. Lett. 73, 2468 (1998).

${ }^{11}$ Gil-Ho Kim, C.-T. Liang, C. F. Huang, J. T. Nicholls, D. A. Ritchie, P. S. Kim, C. H. Oh, J. R. Juang, and Y. H. Chang, Phys. Rev. B 69, 073311 (2004).

${ }^{12}$ B. K. Ridley, Semicond. Sci. Technol. 3, 111 (1988).

${ }^{13}$ M. Russ, C. Meier, B. Marquardt, A. Lorke, D. Reuter, and A. D. Wieck, Phase Transitions 79, 765 (2006)

${ }^{14}$ T. V. Shahbazyan and S. E. Ulloa, Phys. Rev. Lett. 79, 3478 (1997).

${ }^{15}$ E. Ribeiro, E. Muller, T. Heinzel, H. Auderset, K. Ensslin, G. MedeirosRibeiro, and P. M. Petroff, Phys. Rev. B 58, 1506 (1998).

${ }^{16} \mathrm{~A}$. Gold Phys. Rev. B 38, 10798 (1988) 